\title{
Publisher Correction: NCoR/SMRT co-repressors cooperate with c-MYC to create an epigenetic barrier to somatic cell reprogramming
}

Qiang Zhuang, Wenjuan Li, Christina Benda, Zhijian Huang, Tanveer Ahmed, Ping Liu, Xiangpeng Guo, David P. Ibañez, Zhiwei Luo, Meng Zhang, Mazid Md. Abdul, Zhongzhou Yang, Jiayin Yang, Yinghua Huang, Hui Zhang, Dehao Huang, Jianguo Zhou, Xiaofen Zhong, Xihua Zhu, Xiuling Fu, Wenxia Fan, Yulin Liu, Yan Xu, Carl Ward, Muhammad Jadoon Khan, Shahzina Kanwal, Bushra Mirza, Micky D. Tortorella, Hung-Fat Tse, Jiayu Chen, Baoming Qin, Xichen Bao (D),

Shaorong Gao, Andrew P. Hutchins (D) and Miguel A. Esteban (D)

Correction to: Nature Cell Biology https://doi.org/10.1038/s41556-018-0047-x, published online 12 March 2018.

In the version of this Article originally published, in Fig. 2c, the ' + ' sign and 'OSKM' were superimposed in the label '+OSKM'. In Fig. $4 \mathrm{e}$, in the labels, all instances of 'Ant' should have been 'Anti-'. And, in Fig. 7a, the label '0.0' was misplaced; it should have been on the colour scale bar. These figures have now been corrected in the online versions.

Published online: 15 June 2018

https://doi.org/10.1038/s41556-018-0128-x

\section{Publisher Correction: Single-cell multi-omics sequencing of human early embryos}

Lin Li, Fan Guo (D), Yun Gao, Yixin Ren, Peng Yuan, Liying Yan D, Rong Li, Ying Lian, Jingyun Li, Boqiang Hu, Junpeng Gao, Lu Wen, Fuchou Tang (iD and Jie Qiao (iD

Correction to: Nature Cell Biology https://doi.org/10.1038/s41556-018-0123-2, published online 18 June 2018.

In the version of this Resource originally published, owing to a technical error an incorrect file was used for Supplementary Table 2; this has now been replaced with the correct file.

Published online: 18 July 2018

https://doi.org/10.1038/s41556-018-0166-4

\section{Publisher Correction: Tracing the temporal-spatial transcriptome landscapes of the human fetal digestive tract using single-cell RNA-sequencing}

Shuai Gao, Liying Yan D, Rui Wang, Jingyun Li, Jun Yong, Xin Zhou, Yuan Wei, Xinglong Wu, Xiaoye Wang, Xiaoying Fan, Jie Yan, Xu Zhi, Yun Gao, Hongshan Guo, Xiao Jin, Wendong Wang, Yunuo Mao, Fengchao Wang, Lu Wen, Wei Fu,

Hao Ge D, Jie Qiao (D) and Fuchou Tang (D)

Correction to: Nature Cell Biology https://doi.org/10.1038/s41556-018-0105-4, published online 25 May 2018.

In the PDF version of this Resource originally published, on the author list the superscript number 9, to indicate Rui Wang was an equally contributing author, was missing owing to a technical error. This has now been amended. In addition, the authors wish to replace Supplementary Table 2, as in the original version Group 1 immune cells were mis-classified into Group 46 immune cells, resulting in three groups of immune cells where there should have been four. Supplementary Table 2 has now been replaced. 